\title{
BMJ Open Relationships between functional fitness and cognitive impairment in Chinese community-dwelling older adults: a cross-sectional study
}

Mei Yang, ${ }^{1,2}$ Yan Guo, ${ }^{3}$ Jie Gong, ${ }^{3}$ Mengyao Deng, ${ }^{1,2}$ Niannian Yang, ${ }^{3}$ Yaqiong Yan $^{3}$

To cite: Yang M, Guo Y, Gong J, et al. Relationships between functional fitness and cognitive impairment in Chinese community-dwelling older adults: a crosssectional study. BMJ Open 2018;8:e020695. doi:10.1136/ bmjopen-2017-020695

- Prepublication history for this paper is available online. To view these files, please visit the journal online (http://dx.doi. org/10.1136/bmjopen-2017020695).

MY and YG contributed equally.

Received 17 November 2017

Revised 9 March 2018

Accepted 20 April 2018

\section{Check for updates}

${ }^{1}$ Department of Maternal and Child Health, School of Public Health, Wuhan University of Science and Technology, Wuhan, China

${ }^{2}$ Hubei Province Key Laboratory of Occupational Hazard Identification and Control, Wuhan University of Science and Technology, Wuhan, China

${ }^{3}$ Chronic disease prevention and control, Wuhan Centers for Disease Prevention and Control, Wuhan, China

Correspondence to

Dr Yaqiong Yan;

yancdc66@126.com

\section{ABSTRACT}

Objective The aim of this study was to investigate the associations between each functional fitness (FF) domain and cognitive impairment $(\mathrm{Cl})$ in Chinese communitydwelling older adults.

Design A community-based, cross-sectional study was conducted.

Setting Participants were selected by multistage stratified random sampling in Wuhan City, Hubei Province, Central China, during December 2015-May 2016.

Participants A total of 2096 (1031 male and 1065 female) adults older than 65 years were included in our study. Exclusion criteria were age $<65$ years, losing selfliving ability, previously diagnosed with dementia by a neurological physician, severe physical pain, congestive heart failure, dizziness and uncontrolled hypertension (exceeding 160/100 $\mathrm{mm} \mathrm{Hg}$ ).

Primary and secondary outcome measures The Senior Fitness Test and the Mini-Mental State Examination were used to measure FF (including $30 \mathrm{~s}$ chair stand, $30 \mathrm{~s}$ arm curl, 2 min step, 8 foot up-and-go, chair sit-and-reach and back scratch) and screen $\mathrm{Cl}$, respectively. Activities of daily living and instrumental activities of daily living questionnaires were administered to evaluate functional status (FS).

Results $32.16 \%$ were classified as the $\mathrm{Cl}$ group. The results showed that the $\mathrm{Cl}$ group had significantly lower frequency of $30 \mathrm{~s}$ chair stand, $30 \mathrm{~s}$ arm curl and $2 \mathrm{~min}$ step, and longer time to complete the 8 foot up-and-go, shorter chair sit-and-reach and back scratch distance than the non-Cl adults $(p<0.05)$. Except for back scratch, older adults with moderate and high levels of FF were less likely to have $\mathrm{Cl}$ than those with low levels, adjusted by sociodemographics, chronic disease, health condition, health behaviour and $\mathrm{FS}(\mathrm{p}<0.05)$.

Conclusions The relationship between $\mathrm{FF}$ and $\mathrm{Cl}$ was independent of FS decline in Chinese community-dwelling older people.

\section{INTRODUCTION}

Because of the elongation of lifespan and the decline in birth rate, ageing crisis is spreading worldwide. In China, there were already 131.61 million adults older than 65 years old in 2015. Numerous problems are breaking
Strengths and limitations of this study

This is a community-based study with a large sample size.

- The relationships between functional fitness (FF) and cognitive impairment (Cl) were adjusted by potential confounders including disabilities in activities of daily living and instrumental activities of daily living.

- The study did not explore the weight of each FF domain, which might influence the effect of physical exercise.

- The study could not clarify the causality between FF and $\mathrm{Cl}$ or decline in functional status.

out accompanying the ageing process, and dementia has become one of the burdensome public health issues. ${ }^{12}$ As projected by Alzheimer's Disease International in 2015, the number of dementia worldwide was 46.8 million in 2015 and is expected to double every 20 years and arrive at 131.5 million by $2050 .^{3}$

Early identification and intervention have been suggested as the most cost-effective measures to prevent or slow the onset of dementia. ${ }^{4}$ Hence in the past few decades, the focus has shifted from dementia to its preclinical stages such as cognitive impairment (CI). CI is characterised by declines in memory, attention and cognitive function, which greatly affect the quality of life. A previous study has reported that $15.2 \%$ of adults aged $\geq 60$ years were diagnosed with $\mathrm{CI}$ in a Chinese population. ${ }^{5} \mathrm{CI}$ can dramatically increase the burden of family and social service. ${ }^{6} 7$ With the extension of working time and time after retirement, the expectation of older people being 'functional' is increasing. A study to reveal the associations between modifiable factors and CI in older adults is beneficial to develop evidence-based 
programmes to increase the possibility of independent living.

Physical activity is one of the well-established lifestyle factors to maintain cognition. In healthy older people, higher physical activity is associated with higher cognitive performance and lower dementia or CI risk in later life. ${ }^{89}$ Functional fitness (FF), which can be considered as a quantitative indicator of long-term exercise effectiveness, is defined as the physical capacity to perform normal daily activities, including muscle strength, aerobic endurance, flexibility, dynamic balance and agility. Various FF domains can be improved by regular physical activity. Hence, it is reasonable to hypothesise that there may be a relationship between FF and cognitive function in older population. Few studies have been conducted and the results show that the decline in FF precedes the onset of CI. ${ }^{10}{ }^{11}$ However, the risk factors of CI are significantly different between different genetic backgrounds and sociodemographic characteristics such as dietary pattern, economic conditions and so on. ${ }^{12}$ But in Chinese older adults, the relationships between FF and CI are rarely explored.

Functional status (FS), which can be measured by activities of daily living (ADL) and instrumental activities of daily living (IADL) questionnaires, is defined as the ability to perform the basic and instrumental activities of daily living. Existing literatures show that FS decline is consistently related to $\mathrm{CI}$ and is one of the most manifest features in the clinical course of dementia. ${ }^{13-15}$ FS decline has also been proven to be a predictor of CI in older people. ${ }^{16}$ Furthermore, regular exercise may play an important role as a non-pharmacological way to combat FS decline in older people, and there is an interaction between $\mathrm{FF}$ and FS decline. ${ }^{17-19}$ Therefore, it seems possible that FS decline may influence the association between $\mathrm{FF}$ and $\mathrm{CI}$ in older adults. But few studies have considered FS as a potential confounding factor when calculating the association between FF and CI.

Thus, two aims were addressed in our study: (1) to explore the performance level of each $\mathrm{FF}$ domain in older adults with and without CI; and (2) to examine whether the relationships between FF and CI were independent of FS decline in Chinese community-dwelling older people.

\section{METHODS}

\section{Study population}

Our study was designed as a cross-sectional study based in the community. Adults older than 65 years were selected by multistage stratified random sampling in Wuhan City, Hubei Province, Central China, during December 2015-May 2016. According to the census data, 12.36\% were older than 65 years old in Wuhan City. Assuming a design effect of 2, we estimated that a sample size of 1848 would result in a $95 \%$ confidence interval of $1.4 \%$ precision. In consideration of sampling error, denied access and other confounding factors, 2150 subjects were visited. First, seven districts were randomly selected from the 17 districts of Wuhan City. Then, in each district, three to five communities were randomly enrolled, and for each community 60-100 subjects were enrolled. All participants self-reported their medical history and demographic characteristics (gender, age, height, weight, smoking status, years of education and exercise intensity). Exclusion criteria were age $<65$ years, losing self-living ability, previously diagnosed with dementia by a neurological physician, severe physical pain, congestive heart failure, dizziness and uncontrolled hypertension (exceeding 160/100 mm Hg). Finally, a total of 2096 (response rate: 97.49\%, 1031 male and 1065 female) adults were included in our study.

\section{Measurements}

Senior Fitness Test

FF was calculated by the Serious Fitness Test (SFT), which consisted of six items. The SFT is a widely used and reliable test for assessing FF in older people. After $10 \mathrm{~min}$ of warm-up, participants were instructed by the trained instructors. The SFT was then completed in the following order: (1) $30 \mathrm{~s}$ chair stand test to assess lower body muscle strength; (2) 30s arm curl test to assess upper body muscle strength; (3) back scratch test to assess upper body (shoulder) flexibility; (4) chair sit-and-reach test to assess lower body flexibility; (5) 8 foot up-and-go test to assess agility and dynamic balance as an index of basic mobility skills; and (6) 2 min step test to assess aerobic endurance. Each test was strictly performed according to the SFT manual. ${ }^{20}$

For each domain, the participants were classified into low fitness (lower than the lower quartile), moderate fitness (between the lower and upper quartiles) or high fitness (higher than the upper quartile).

\section{Physical Activity Scale for the Elderly}

The Physical Activity Scale for the Elderly (PASE) is a brief, easily scored, reliable and valid instrument for the assessment of physical activity in epidemiology studies of older people. This scale comprised self-reported occupational, household and leisure items over a 1-week period. The participants were required to report the number of days per week and the number of hours per day for each type of activities. Then PASE scores were calculated from weights and frequency values for each item. The PASE score ranged 0 360 points and a higher score represented a greater amount of physical activity. ${ }^{21}$

\section{MMSE measurement}

The Mini-Mental State Examination (MMSE), which had been proven as a reliable, sensitive and valid method, was used to screen CI. The Chinese version of the MMSE was performed by trained nurses. The MMSE measured the cognitive level of older adults in terms of (1) temporal and spatial orientation: 10 points; (2) immediate memory: 3 points; (3) recall: 3 points; (4) attention: 5 points; and (5) language: 9 points. The MMSE score ranged $0 \sim 30$ points, where a higher score indicated a better cognition level. In 
the Chinese population, it was suggested that CI should be defined as MMSE score $<24$ in people with education of less than 9 years, or $<26$ in those who received formal education for more than 9 years. ${ }^{22}$

\section{$\mathrm{ADL}$ and IADL}

FS could be measured by ADL and IADL questionnaires. ADL included six items (rising or lying down, feeding, dressing, bathing, toileting, and urinary and faecal continence), and IADLs included seven items (using a telephone, shopping, doing housework, cooking, using transportation, taking medications and handling finances). Both questionnaires were obtained directly from the subjects without $\mathrm{CI}$ and from a close relative of the subjects with CI. ADL or IADL disability was defined as dependence in at least one item of ADL or IADL, respectively.

\section{Patient and public involvement}

Through multistage stratified random sampling, the older adults were selected in the communities. After obtaining informed consent, the trained community doctors in each selected community were responsible for investigating the subjects, and the results of measurements would be disseminated to participants immediately after the investigation was completed.

\section{Statistical analysis}

Normality of distribution for continuous variables was tested by the Kolmogorov-Smirnov test. Normal distribution data were presented as mean $\pm \mathrm{SD}$, and the differences among groups were compared by unpaired Student's t-test or analysis of variance. For qualitative data, $\chi^{2}$ tests were performed for comparison between groups. Pearson's correlation coefficients were conducted between PASE score and FF. Logistic regression was performed to evaluate the associations between FF domains and CI. ORs with $95 \%$ confidence intervals, adjusted by sociodemographics, chronic disease, health condition, health behaviour and FS, were calculated. $\mathrm{P}<0.05$ was accepted as statistically significant. Analyses were performed with SAS V.9.4 software for Windows.

\section{RESULTS}

\section{Baseline characteristics}

Among the 2096 subjects, $32.16 \%$ were classified as CI. The characteristics of older adults with and without CI are summarised in table 1. Compared with the non-CI group, the CI group had a higher age and female proportion, but lower height, weight, body mass index (BMI), waist circumference and PASE score $(\mathrm{p}<0.05)$. The distribution was significantly different in falls within 1 year, smoking, regular exercise, PASE score and hyperlipidaemia between the CI and non-CI groups $(p<0.05)$. Furthermore, the rate of ADL disability was significantly higher in the CI group $\left(11.31 \%\right.$ vs $5.76 \% ; \chi^{2}=25.95$, $\mathrm{p}<0.0001)$, so was IADL disability $(66.47 \%$ vs $20.70 \%$; $\left.\chi^{2}=418.41, \mathrm{p}<0.0001\right)$. The older adults with CI had significantly lower frequency of $30 \mathrm{~s}$ chair stand, $30 \mathrm{~s}$ arm curl and $2 \mathrm{~min}$ step, and longer time to complete the 8 foot up-and-go, shorter chair sit-and-reach and back scratch distance than the non-CI group $(\mathrm{p}<0.05)$. In addition, as presented in table 2, PASE score was significantly correlated with each FF domain $(\mathrm{p}<0.05)$.

\section{Associations between $\mathrm{FF}$ and $\mathrm{Cl}$}

Table 3 presents the adjusted ORs for CI risk according to FF levels. Using sociodemographic characteristics (including age, sex, education years, BMI and falls within 1year), chronic disease (including diabetes, hypertension, hyperlipemia and coronary artery disease), health behaviour (including smoking, regular exercise and PASE score) and FS (ADL disability and IADL disability) as covariates, except for back scratch, older adults with moderate or high levels of $30 \mathrm{~s}$ chair stand, chair sit-andreach, $30 \mathrm{~s}$ arm curl, 2 min step and 8 foot up-and-go were less likely to have CI than those with low levels.

\section{DISCUSSION}

As far as we know, this is the first study that examined the relationships between each FF domain and CI among Chinese older community-dwelling adults. The key results of this study showed that the prevalence of CI was $32.16 \%$, and except for back scratch, five other FF domains were significantly independently correlated with CI risk.

With the same screening tool (MMSE) and diagnostic threshold, Lin $e t a l^{23}$ reported that $18.6 \%$ of older male Taiwanese were found to have CI, which was similar to our study (19.88\% in male). In addition, existing research showed that women were at higher risk of having CI than men, ${ }^{24}$ and in our study as much as $42.72 \%$ female older adults were classified to have CI. Using the Abbreviated Mental Test and the Progressive Forgetfulness Questionnaire, Hilal et a $\tilde{l}^{\tilde{l}}$ concluded that $15.2 \%$ of Chinese subjects (aged $\geq 60$ years) were diagnosed with CI in a Singapore study, and in Western countries and Korea the prevalence of CI was $1.7 \%-22.5 \%{ }^{25}$ and $24.1 \%,{ }^{26}$ respectively. All reported data were lower than our results. Evaluation criterion varied between different cognitive screening tools and this would affect the judgement of CI. Moreover, the prevalence of CI was also associated with the composition of subjects, such as age, ${ }^{5}$ educational level, ${ }^{23}$ marital status ${ }^{27}$ and so on; hence, more rigorous survey should be conducted to confirm the prevalence of CI in Chinese community-dwelling population.

Overall, our results, which presented a significant association between poor $\mathrm{FF}$ and poor cognitive function in older adults, were consistent with previous studies. ${ }^{28} 29$ In addition, we stratified and analysed the association between FF and CI by men, women and IADL disability (the sample size for ADL disability was insufficient for stratified analysis), and the results also showed that older adults with moderate or high levels of FF were less likely to have CI than those with low levels for each subgroup 
Open Access

Table 1 Characteristics of the community-dwelling older adults with and without $\mathrm{Cl}$

\begin{tabular}{|c|c|c|c|}
\hline & $\mathrm{Cl}(\mathrm{n}=674)$ & Non-Cl $(n=1422)$ & $P$ values \\
\hline \multicolumn{4}{|l|}{ Sociodemographic characteristics } \\
\hline Age (years) & $74.40 \pm 5.73$ & $71.79 \pm 4.63$ & $<0.0001$ \\
\hline Female, n (\%) & $457(67.80)$ & $608(42.76)$ & $<0.0001$ \\
\hline Education >9years, n (\%) & $60(8.90)$ & $368(27.18)$ & $<0.0001$ \\
\hline Height (cm) & $156.30 \pm 7.83$ & $161.70 \pm 8.01$ & $<0.0001$ \\
\hline Weight (kg) & $58.93 \pm 10.51$ & $64.53 \pm 10.68$ & $<0.0001$ \\
\hline $\mathrm{BMI}\left(\mathrm{kg} / \mathrm{m}^{2}\right)$ & $24.07 \pm 3.64$ & $24.66 \pm 3.59$ & $<0.001$ \\
\hline Waist circumference $(\mathrm{cm})$ & $86.63 \pm 10.04$ & $88.20 \pm 9.75$ & $<0.001$ \\
\hline Falls within 1 year, $\mathrm{n}(\%)$ & $99(14.69)$ & $138(9.73)$ & $<0.01$ \\
\hline \multicolumn{4}{|l|}{ Chronic disease } \\
\hline Diabetes (yes), n (\%) & 90 (13.35) & 199 (13.99) & 0.69 \\
\hline Hypertension (yes), n (\%) & $383(56.99)$ & $762(53.62)$ & 0.15 \\
\hline Hyperlidaemia (yes), n (\%) & $50(7.42)$ & $164(11.53)$ & $<0.01$ \\
\hline Coronary artery disease (yes), n (\%) & $93(13.80)$ & $181(12.73)$ & 0.50 \\
\hline \multicolumn{4}{|l|}{ Health behaviours } \\
\hline Smoking (yes), n (\%) & $88(13.06)$ & $289(20.32)$ & $<0.0001$ \\
\hline Regular exercise (yes), n (\%) & $396(56.03)$ & $1115(80.04)$ & $<0.0001$ \\
\hline PASE & $112.93 \pm 67.60$ & $144.78 \pm 67.72$ & $<0.0001$ \\
\hline \multicolumn{4}{|l|}{ Functional status } \\
\hline ADL disability (yes), n (\%) & $76(11.31)$ & $73(5.76)$ & $<0.0001$ \\
\hline IADL disability (yes), $\mathrm{n}(\%)$ & $448(66.47)$ & $294(20.70)$ & $<0.0001$ \\
\hline \multicolumn{4}{|l|}{ Functional fitness } \\
\hline 30 s chair stand $(n)$ & $11.69 \pm 5.46$ & $14.42 \pm 6.02$ & $<0.0001$ \\
\hline Chair sit-and-reach $(\mathrm{cm})$ & $-5.80 \pm 9.93$ & $-2.97 \pm 8.43$ & $<0.0001$ \\
\hline $30 \mathrm{~s}$ arm curl (n) & $13.66 \pm 6.93$ & $18.59 \pm 9.39$ & $<0.0001$ \\
\hline 2 min step (n) & $64.03 \pm 37.10$ & $82.37 \pm 37.53$ & $<0.0001$ \\
\hline Back scratch $(\mathrm{cm})$ & $-15.61 \pm 12.29$ & $-11.81 \pm 12.24$ & $<0.0001$ \\
\hline 8 foot up-and-go (s) & $10.37 \pm 4.58$ & $8.61 \pm 4.10$ & $<0.0001$ \\
\hline
\end{tabular}

$A D L$, activities of daily living; BMI, body mass index; $\mathrm{Cl}$, cognitive impairment; IADL, instrumental activities of daily living; PASE, Physical Activity Scale for the Elderly.

(data not shown). For each FF domain, we found that in the Chinese population, lower and upper muscle strength, lower body flexibility, dynamic balance and aerobic endurance were independently associated with cognitive function, but not upper body flexibility. Hesseberg et $a l^{11}$ also concluded that there were significant associations between the components of physical fitness and cognition, except flexibility in Norwegians. ${ }^{11}$ And in the Epidemiologie del' Osteoporose (EPIDOS) cohort study, researchers revealed that poor dynamic balance in

Table 2 Correlation between PASE score and functional fitness

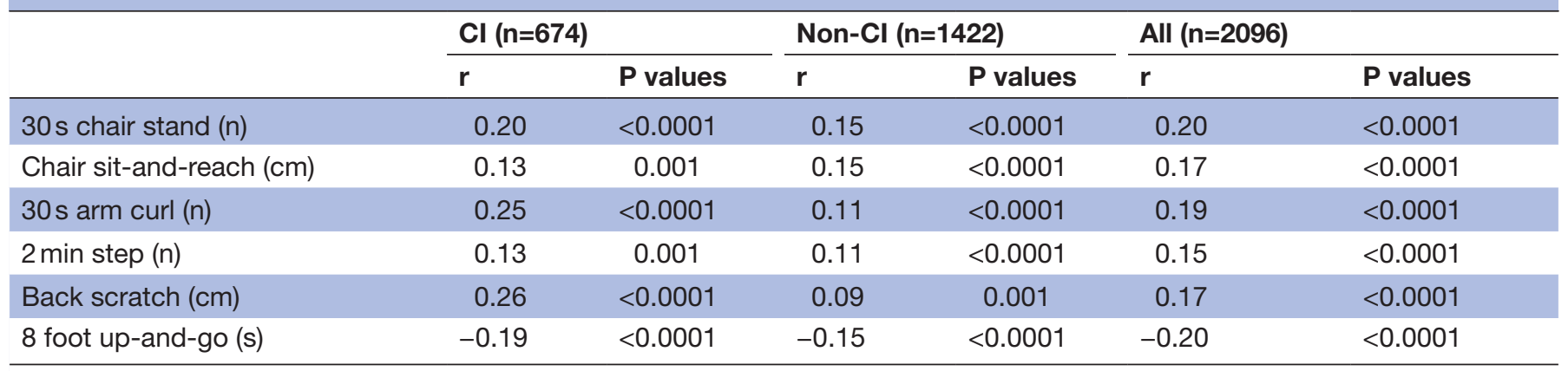

$\mathrm{Cl}$, cognitive impairment; PASE, Physical Activity Scale for the Elderly. 
Table 3 Adjusted OR for cognitive impairment according to function fitness levels

\begin{tabular}{|c|c|c|c|c|c|}
\hline & Low & Moderate & P values* & High & P values* \\
\hline 30 s chair stand & 1.00 & 0.76 (0.59 to 0.97$)$ & 0.03 & $0.49(0.35$ to 0.67$)$ & $<0.0001$ \\
\hline Chair sit-and-reach & 1.00 & 0.70 (0.54 to 0.90$)$ & 0.01 & 0.53 (0.38 to 0.73$)$ & $<0.001$ \\
\hline 2 min step & 1.00 & 0.72 (0.56 to 0.93$)$ & 0.01 & 0.51 (0.37 to 0.70$)$ & $<0.0001$ \\
\hline Back scratch & 1.00 & 1.07 (0.83 to 1.38$)$ & 0.60 & $0.90(0.66$ to 1.24$)$ & 0.53 \\
\hline
\end{tabular}

*Adjusted by sociodemographic, health condition, health behaviour and functional status.

women, which was assessed by five-time-sit-to-stand test, was associated with a higher risk of CI. ${ }^{30}$ Moreover, it is well known that FF was positively associated with physical activity, and our results showed that a greater amount of physical activity was positively correlated with each FF domain. But different types of activity might have different effects on each domain. For example, square-stepping could improve balance and yoga was as good as stretching-strengthening exercises. ${ }^{31} 32$ Until now, little is known about the mechanisms between FF and cognitive function. But FF test required refined brain control for the initiation of tasks, recruitment of muscles and motor coordination. Hence, the concurrent deterioration of the brain regions which are responsible for cognitive and physical performance might be one possible mechanism explaining the observed association. ${ }^{33}{ }^{34}$ Moreover, angiogenesis, neurogenesis and synaptogenesis might also play an important role, but further clinical and laboratory investigations need to be conducted to confirm this. ${ }^{34} 35$

\section{Limitations}

Even with a large sample size and adjustment of potential confounders, our study had some limitations. First, although the results showed that five domains of FF were independently associated with CI, our study did not explore the weight of each domain. Second, this study was a cross-sectional study which could not clarify the causality between FF and CI or FS decline. Third, except for FS, there were other confounding factors, such as nutrition status and so on.

\section{Future directions}

Taking these limitations into account, further larger and prospective research is needed to explore the association between FF and CI in Chinese older populations. Furthermore, the most effective type and intensity of physical exercise that aims to improve cognitive performance in older adults should be investigated.

In summary, the present study concluded that the prevalence of MMSE-based CI was $32.16 \%$. In addition, lower frequency of $30 \mathrm{~s}$ chair stand, $30 \mathrm{~s}$ arm curl and $2 \mathrm{~min}$ step, and longer time to complete the 8 foot up-and-go and shorter chair sit-and-reach were significantly associated with CI, independent of confounding factors including sociodemographics, health behaviour, ADL disability and IADL disability. This suggested that physical exercise aimed to maintain or promote the above-mentioned fitness might be an effective element of a healthy lifestyle strategy to prevent CI in Chinese older populations.

Contributors All authors contributed significantly to this work. YG and MY are joint first authors. YG obtained funding. YG, MY and YY conceived and designed the experiments. YG, JG, MD and NY performed the experiments. YG and MY analysed the data. MY wrote the paper. All authors have read and approved the final manuscript.

Funding This work was supported by a grant from the Wuhan Health and Family Planning Commission (WG14A02).

Competing interests None declared.

Patient consent Not required.

Ethics approval This study was approved by the Ethical Review Committee of the Wuhan Centers for Disease Control and Prevention.

Provenance and peer review Not commissioned; externally peer reviewed.

Data sharing statement № additional data are available.

Open Access This is an Open Access article distributed in accordance with the Creative Commons Attribution Non Commercial (CC BY-NC 4.0) license, which permits others to distribute, remix, adapt, build upon this work non-commercially, and license their derivative works on different terms, provided the original work is properly cited and the use is non-commercial. See: http://creativecommons.org/ licenses/by-nc/4.0/

(c) Article author(s) (or their employer(s) unless otherwise stated in the text of the article) 2018. All rights reserved. No commercial use is permitted unless otherwise expressly granted.

\section{REFERENCES}

1. Ballard C, Gauthier S, Corbett A, et al. Alzheimer's disease. Lancet 2011;377:1019-31.

2. Panza F, D'Introno A, Colacicco AM, et al. Current epidemiology of mild cognitive impairment and other predementia syndromes. Am J Geriatr Psychiatry 2005;13:633-44.

3. Prince M, Wimo A, Guerchet M, et al. World Alzheimer report 2015. The global impact of dementia. An Analysis of Prevalence, Incidence, Cost and Trends 2015.

4. Sperling RA, Aisen PS, Beckett LA, et al. Toward defining the preclinical stages of Alzheimer's disease: recommendations from the National Institute on Aging-Alzheimer's Association workgroups on diagnostic guidelines for Alzheimer's disease. Alzheimers Dement 2011;7:280-92.

5. Hilal S, Ikram MK, Saini M, et al. Prevalence of cognitive impairment in Chinese: epidemiology of dementia in Singapore study. J Neurol Neurosurg Psychiatry 2013;84:686-92.

6. Torres HA, Kabir ZN, Winblad B. Distribution of home help services in an elderly urban population: Data from the kungsholmen project. Scand J Soc Welf 1995;4:274-9.

7. Agüero-Torres $\mathrm{H}$, von Strauss $\mathrm{E}$, Viitanen $\mathrm{M}$, et al. Institutionalization in the elderly: the role of chronic diseases and dementia. Crosssectional and longitudinal data from a population-based study. J Clin Epidemiol 2001;54:795-801. 
8. Bherer L, Erickson KI, Liu-Ambrose T. A review of the effects of physical activity and exercise on cognitive and brain functions in older adults. J Aging Res 2013;2013:1-8.

9. Blondell SJ, Hammersley-Mather R, Veerman JL. Does physical activity prevent cognitive decline and dementia?: A systematic review and meta-analysis of longitudinal studies. BMC Public Health 2014;14:510.

10. Verghese J, Lipton RB, Hall CB, et al. Abnormality of gait as a predictor of non-Alzheimer's dementia. N Engl J Med 2002;347:1761-8.

11. Hesseberg K, Bentzen $\mathrm{H}$, Ranhoff $\mathrm{AH}$, et al. Physical fitness in older people with mild cognitive impairment and dementia. J Aging Phys Act 2016;24:92-100.

12. Allès $B$, Samieri $C$, Féart $C$, et al. Dietary patterns: a novel approach to examine the link between nutrition and cognitive function in older individuals. Nutr Res Rev 2012;25:207-22.

13. De Vriendt $P$, Mets $T$, Petrovic M, et al. Discriminative power of the advanced activities of daily living (a-ADL) tool in the diagnosis of mild cognitive impairment in an older population. Int Psychogeriatr 2015;27:1419-27.

14. Royall DR. Mild cognitive impairment and functional status. J Am Geriatr Soc 2006;54:163-5.

15. Royall DR, Lauterbach EC, Kaufer D, et al. The cognitive correlates of functional status: a review from the Committee on Research of the American Neuropsychiatric Association. J Neuropsychiatry Clin Neurosci 2007:19:249-65.

16. Chodosh J, Miller-Martinez D, Aneshensel CS, et al. Depressive symptoms, chronic diseases, and physical disabilities as predictors of cognitive functioning trajectories in older Americans. J Am Geriatr Soc 2010;58:2350-7.

17. Nagamatsu T, Oida Y, Kitabatake Y, et al. A 6-year cohort study on relationship between functional fitness and impairment of ADL in community-dwelling older persons. J Epidemiol 2003;13:142-8.

18. Ogliari G, Sabayan B, Mari D, et al. Age- and functional statusdependent association between blood pressure and cognition: the milan geriatrics 75+ cohort study. J Am Geriatr Soc 2015;63:1741-8.

19. Ueya K, Koyama S. Relationship among Physical Fitness, ADL, QOL, and Actual Conditions of Daily Life of the Elderly Persons Concerning the New Physical Fitness Test of Japanese Ministry of Education and Science. Bulletin of Teikyo University of Science \& Technology 2011;7:25-34.

20. Rikli RE, Jones CJ. Senior fitness test manual. J Aging Phys Act 2012;10:110-1.

21. Washburn RA, Smith KW, Jette AM, et al. The Physical Activity Scale for the Elderly (PASE): development and evaluation. J Clin Epidemiol 1993;46:153-62.
22. Guo NW, Liu HC, Wong PF. Chinese version and norms of the MiniMental State Examination. Biomicrofluidics 1988;8:44107.

23. Lin CS, Lin MH, Peng LN, et al. Screening cognitive impairment among institutionalized older Chinese men in Taiwan: a new minimum data set-based dementia screening tool is needed. Arch Gerontol Geriatr 2011;53:e25-e28.

24. Zhang Z. Gender differentials in cognitive impairment and decline of the oldest old in China. J Gerontol B Psychol Sci Soc Sci 2006;61:S107-S115.

25. Luck T, Luppa M, Briel S, et al. Incidence of mild cognitive impairment: a systematic review. Dement Geriatr Cogn Disord 2010;29:164-75.

26. Kim KW, Park JH, Kim MH, et al. A nationwide survey on the prevalence of dementia and mild cognitive impairment in South Korea. J Alzheimers Dis 2011;23:281-91.

27. Feng L, $\mathrm{Ng} \mathrm{XT}$, Yap P, et al. Marital status and cognitive impairment among community-dwelling chinese older adults: The role of gender and social engagement. Dement Geriatr Cogn Dis Extra 2014;4:375-84

28. Lin PS, Hsieh CC, Cheng HS, et al. Association between physical fitness and successful aging in taiwanese older adults. PLoS One 2016;11:e150389.

29. Lee S, Han J, Jin Y, et al. Poor physical fitness is independently associated with mild cognitive impairment in elderly Koreans. Biol Sport 2016:33:57-62.

30. Annweiler C, Schott AM, Abellan van Kan G, et al. The fivetimes-sit-to-stand test, a marker of global cognitive functioning among community-dwelling older women. J Nutr Health Aging 2011:15:271-6.

31. Teixeira CV, Gobbi S, Pereira JR, et al. Effect of square-stepping exercise and basic exercises on functional fitness of older adults. Geriatr Gerontol Int 2013;13:842-8.

32. Gothe NP, McAuley E. Yoga is as good as stretching-strengthening exercises in improving functional fitness outcomes: results from a randomized controlled trial. J Gerontol A Biol Sci Med Sci 2016:71:406-11.

33. Resnick SM, Pham DL, Kraut MA, et al. Longitudinal magnetic resonance imaging studies of older adults: a shrinking brain. $J$ Neurosci 2003;23:3295-301.

34. Salat $\mathrm{DH}$. The declining infrastructure of the aging brain. Brain Connect 2011;1:279-93.

35. Swain RA, Harris AB, Wiener EC, et al. Prolonged exercise induces angiogenesis and increases cerebral blood volume in primary motor cortex of the rat. Neuroscience 2003;117:1037-46. 